\title{
Is Auditory Steady-State Response Testing the Key for Diagnosing Non-Organic Hearing Disorders? Implications for Current Audiological Practice
}

\author{
John Plioutas ${ }^{1 *}$, Petros V. Vlastarakos ${ }^{2 *}$, Alexandros Delidis ${ }^{1,3}$, Alexandra Vasileiou ${ }^{4}$, \\ Thomas P. Nikolopoulos ${ }^{1,3}$, and Pavlos Maragoudakis ${ }^{1,3}$ \\ ${ }^{1}$ Department of Otorhinolaryngology, ${ }^{4}$ Clinic of Pediatric Hearing Loss, Attikon University Hospital, Athens, Greece \\ 2Department of Otorhinolaryngology, MITERA Paediatric Infirmary, Athens, Greece \\ ${ }^{3}$ Department of Otorhinolaryngology, University of Athens, Athens, Greece
}

Received April 25, 2021

Revised July 11,2021

Accepted October 21, 2021

Address for correspondence

Petros V. Vlastarakos,

$\mathrm{MD}, \mathrm{MSc}, \mathrm{PhD}$, IDO-HNS

Department of Otorhinolaryngology, MITERA Paediatric Infirmary,

6 Erythrou Stavrou Str.,

Marousi-Athens 15123, Greece

Tel +00302114085381

Fax +00302106869161

E-mail pevlast@hotmail.com

*These authors contributed equally to this work.
Background and Objectives: To describe all possible facets of non-organic hearing disorders (NOHD) and emphasize the superiority of auditory steady-state response (ASSR) over previously employed hearing assessment tools. Subjects and Methods: A series of seven patients consisting of three males and four females with NOHD were assessed at Attikon University Hospital (age range: 17-59 years). Three patients had Munchausen syndrome, three intentionally feigned hearing loss, and one intentionally feigned normal hearing. The audiological evaluation consisted of tympanometry, pure-tone audiometry, and ASSR testing. Results: The hearing of all patients was accurately determined using ASSR. The results were confirmed by auditory brainstem responses (ABR) and otoacoustic emissions. Conclusions: NOHD is a multi-faceted condition encompassing various etiologies. ASSR testing represents an objective and reliable method of hearing assessment, which can serve as a gold standard method for distinguishing NOHD from actual hearing loss. It can reliably indicate the hearing levels at the four main frequencies $(500,1,000,2,000$, and 4,000 Hz) by obtaining a valid estimated audiogram through statistical measures. Compared to ABR testing, ASSR thresholds are closer to the actual audiometric thresholds in the presence of hearing impairment and are superior when the corresponding pure-tone audiogram is widely ranging between the adjacent frequencies or when the obtained ABR curves are not easily distinguished. A non-confrontational approach should be adopted by ENT doctors towards cases of suspected NOHD as the use of ASSR could reliably assess hearing even when medical or medico-legal implications are involved.

J Audiol Otol 2022;26(2):61-67

Keywords: Non-organic hearing disorder; Diagnosis; ASSR; Auditory brainstem responses; Malingering.

\section{Introduction}

Deception is an act or statement, which misleads, hides the truth, or promotes a belief, concept, or idea that is not true, usually for personal gain or advantage [1]. Intent is critical with regard to deception, as it differentiates itself from an honest mistake.

This is an Open Access article distributed under the terms of the Creative Commons Attribution Non-Commercial License (https://creativecommons.org/licenses/by-nc/4.0/) which permits unrestricted non-commercial use, distribution, and reproduction in any medium, provided the original work is properly cited.
Deception does not occur frequently in audiology, however, non-organic hearing disorders (NOHD) should be considered in everyday audiological practice. Such disorders may involve otherwise normal individuals, who are seeking personal or economic gains, or people, who exaggerate a mild hearing loss, or pretend to be profoundly deaf in the context of a legal dispute, or even individuals, who have emotional or psychological disorders, and are in seek of attention or constant hospitalization. Cases of alleged hearing adequacy, when this represents a prerequisite for professional evolvement, complete the spectrum of NOHD. 
The assessment of suspected NOHD patients should be prompt, and the methodology employed should comprise testing, which would be quick, adequate, objective, and easily repeated. Auditory steady-state response (ASSR) represents such an objective method of hearing asssessment, which can reliably distinguish NOHD from actual hearing loss in doubtful cases.

The aim of the present case series was to describe all the possible facets of NOHD and emphasize the superiority of ASSR, compared to previously employed hearing assessment tools.

\section{Subjects and Methods}

A series of seven patients with NOHD were assessed at the Audiology Unit of Attikon University Hospital. Three of them were males, and four females. The age of the examinees ranged between 17 and 59 years.

The audiological evaluation comprised tympanometry, pure-tone audiometry, and ASSR testing, and the results were complemented by auditory brainstem responses (ABR), and otoacoustic emissions (OAEs). The research protocol was submitted, and received ethical approval by the Ethics Committee of the University of Athens (IRB approval no: 141501476208/05/2015), prior to commencing data collection. All procedures were in accordance with the 2013 Helsinki declaration. Participants were asked to sign a consent form before being enrolled in the study.

With the patient in a relaxed position in a soundproof chamber, $40 \mathrm{~Hz}$ stimulus rate CE-chirp ${ }^{\circledR}$ sounds are given through insert phones (adult awake protocol). The device used is the Eclipse EP $25^{\mathrm{TM}}$ (Interacoustics, Middelfart, Denmark). The examination time is 6 minutes per examined hearing level (HL). A given stimulus of $20-30 \mathrm{~dB}$ higher than the least threshold in four frequencies of the pure tone audiogram (500, $1,000,2,000$, and $4,000 \mathrm{~Hz}$ ) is presented in both ears at the same time. Whenever, the curve of the stimulus reaches $100 \%$ certainty, it turns green (positive outcome), and the operator lowers the stimulus by $10 \mathrm{~dB}$. If the curve remains under $100 \%$ by the end of the examination time, the outcome is negative. The examiner may choose to extend the testing by $1 \mathrm{~min}-$ ute, if he/she believes that a positive outcome is likely to occur. An estimated audiometric curve is obtained, based on the least threshold stimuli of the four examined frequencies, producing a positive outcome, at the end of the test. The perceived threshold is automatically transformed from $\mathrm{dB}$ sound-pressure level to $\mathrm{dB} \mathrm{HL}$, thus obtaining a reliable estimate of the pure-tone audiogram.

\section{Case descriptions}

\section{Munchausen syndrome}

Munchausen syndrome is a psychological disorder, where a person pretends to be ill, or deliberately produces symptoms of illness in him/herself. The main intent is to assume a "sickness role," which would place the person at the centre of care and attention, without, however, any other practical benefit from this pretense (i.e., claiming an incapacity benefit). Possible causes of Munchausen syndrome include childhood trauma, personality disorders, or antipathy against authority figures or healthcare professionals.

All three patients in the present series were females between the second and third decade of their lives. Two of them presented with acute bilateral deafness, whilst the third initially presented with unilateral, followed by bilateral hearing loss in the following three months. Past medical history revealed irritable bowel syndrome in the two older patients, whilst the socio-environmental living conditions in two of the patients were challenging.

Clinical ENT examination was unremarkable in all cases, and the tympanometric curves were suggestive of normal middle ear ventilation. Audiometric testing was suggestive of severe to profound hearing loss, bilaterally in two cases, and initially unilaterally, followed by contralateral hearing impairment within three months, in the third case (Fig. 1A). However, the non-significant impairment in the communicative abilities of all patients, largely inconsistent with the audiometric curves, along with previous hospital admissions for various reasons, necessitated ASSR assessment, which proved normal in all patients (Fig. 1B). ABR and OAEs were also indicative of normal hearing (albeit not being frequency-specific) (Fig. $1 \mathrm{C}$ and $\mathrm{D}$, respectively). Treatment for sudden sensorineural hearing loss was discontinued in all patients, who were subsequently referred for psychiatric evaluation.

\section{Malingering}

Three patients in the present series intentionally feigned profound hearing loss. These comprised two males and one female patient. The socio-economic background among the three individuals differed. One was a male economic refugee, who sought state benefit, by claiming profound bilateral hearing loss. A second male patient was an ex-soldier claiming compensation by the Greek Military for unilateral deafness, as a result of a grenade explosion. The third patient was a female, who had been receiving state benefit for incapacitation, due to profound bilateral hearing loss, for the past 40 years, and was re-examined, following strong reservations about her hearing loss from the State Otorhinolaryngologist. 
Plioutas J, et al.
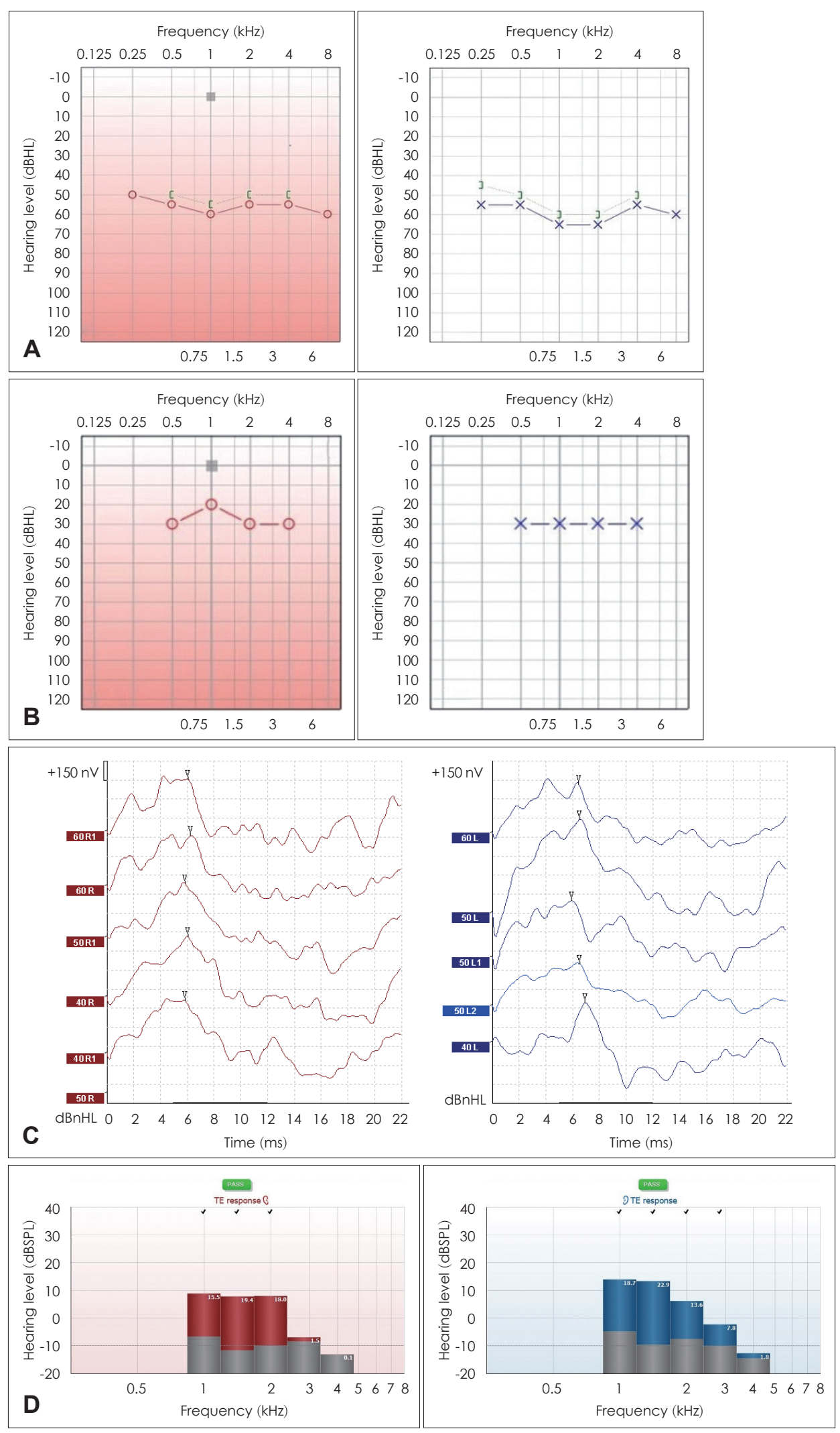

Fig. 1. Comprehensive audiologic testing of a 21-year-old patient with Munchausen syndrome. A: Pure-tone audiogram. B: Estimated auditory steady-state response audiogram, indicative of normal hearing. C, D: auditory brainstem response curves, and otoacoustic emission graph. Easily distinguished wave $\mathrm{V}$ at $40 \mathrm{dBnHL}$, and elicitation of transient evoked otoacoustic emissions, indicative of normal hearing. 

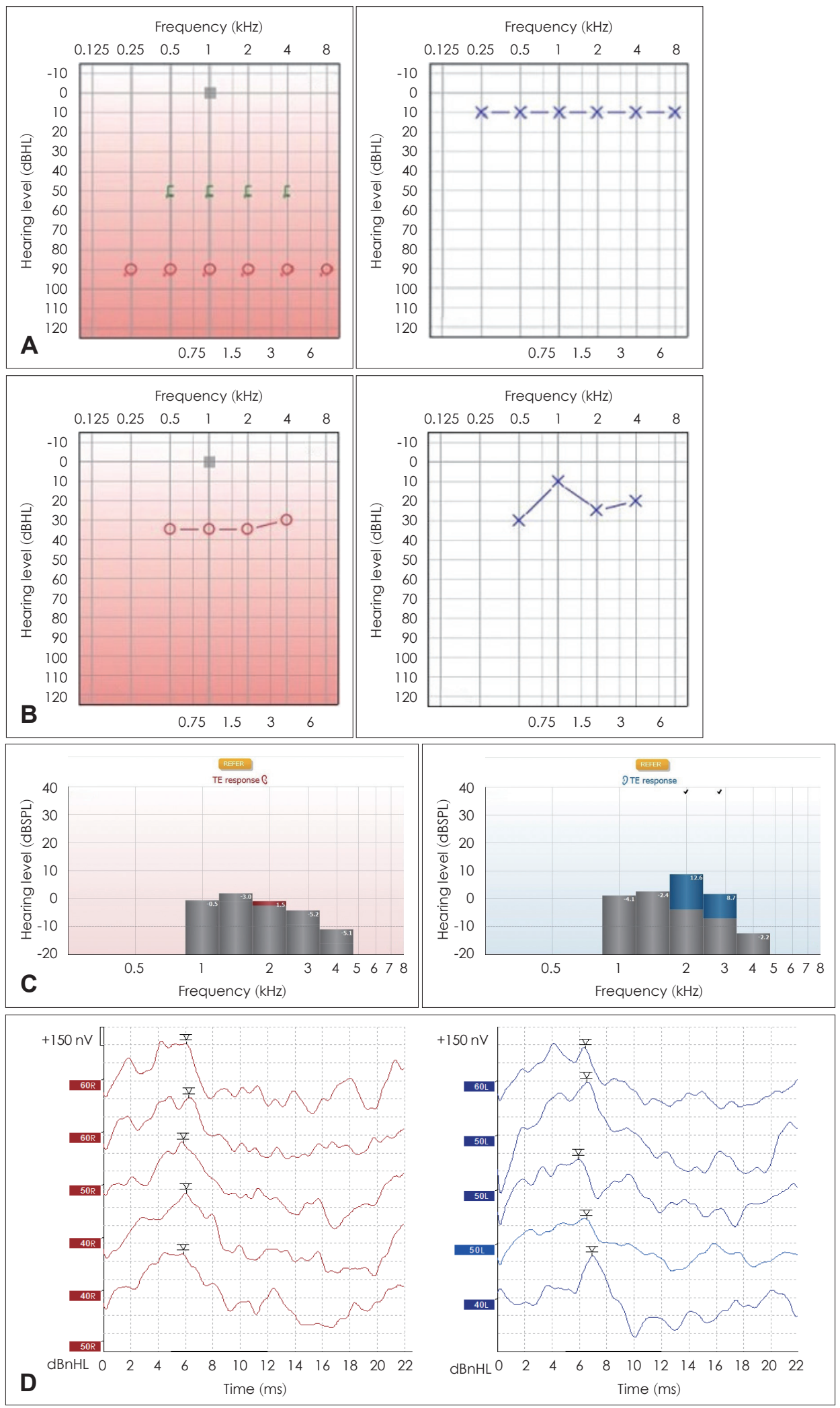

Fig. 2. Comprehensive audiologic testing of a 26-year-old ex-soldier feigning right-sided profound hearing loss, allegedly following a grenade explosion (A, B), and a female patient exaggerating a mild hearing loss, to continue receiving state benefit for incapacitation (C, D). A: Pure-tone audiogram. B: Estimated auditory steady-state response audiogram, indicative of normal hearing. C, D: auditory brainstem response curves, and otoacoustic emission graph. Partial elicitation of transient evoked otoacoustic emissions in the left ear, and easily distinguished wave $\mathrm{V}$ at $50 \mathrm{dBnHL}$ bilaterally. 
Clinical ENT examination was unremarkable in all cases, and the tympanometric curves were suggestive of normal middle ear ventilation. Audiometric testing was suggestive of profound hearing loss, bilaterally in two cases, and unilaterally involving the right ear in the third case (Fig. 2A). Interestingly, the communicative abilities of all patients appeared significantly impaired. ASSR assessment proved normal with regard to the male patients (Fig. 2B), and demonstrated mild hearing
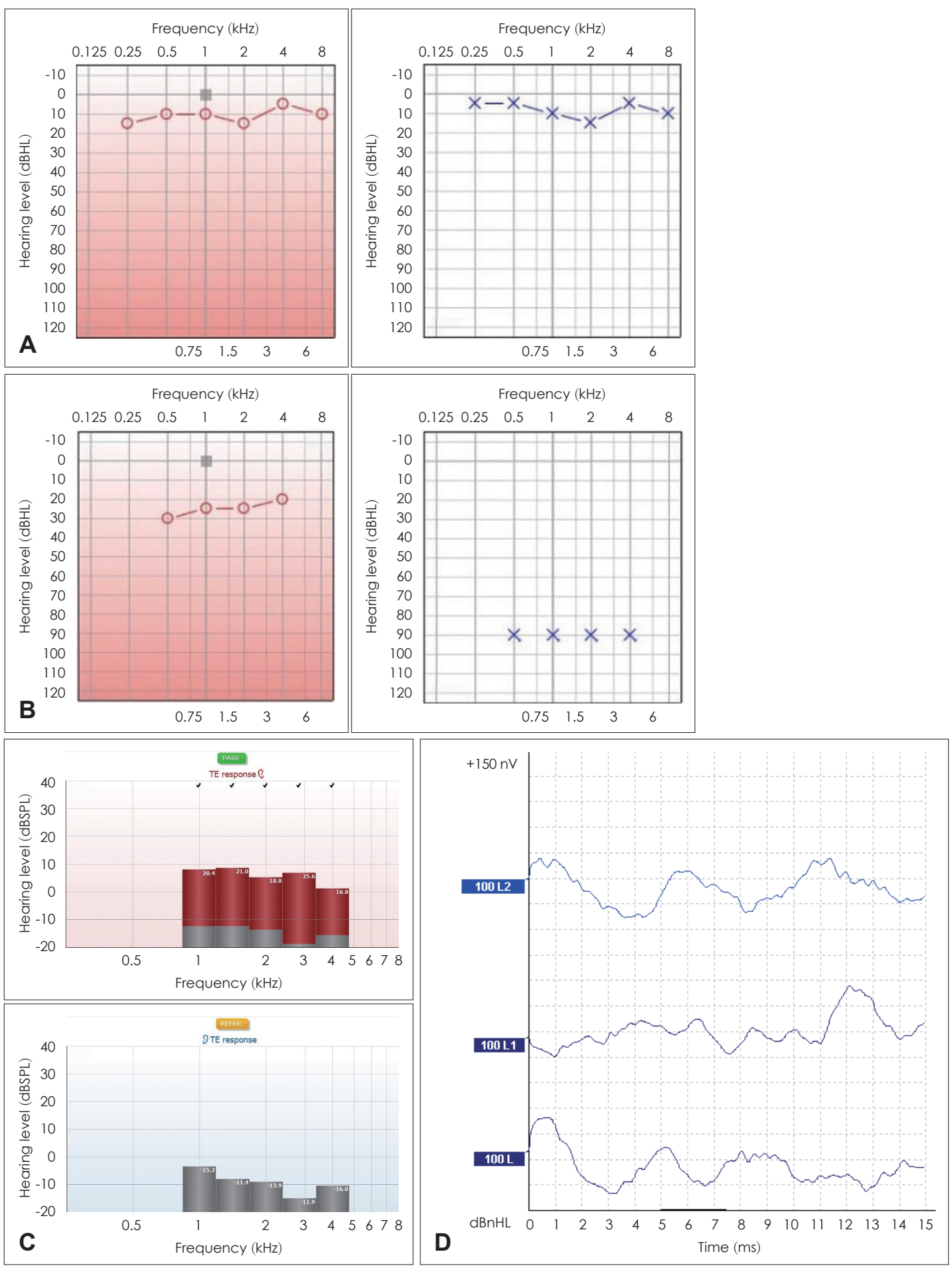

Fig. 3. Comprehensive audiologic testing of a 50-year-old career naval officer claiming normal bilateral hearing. A: Pure-tone audiogram. B: Estimated auditory steady-state response audiogram, indicative of profound left hearing loss. C, D: auditory brainstem response curves, and otoacoustic emission graph. Unilateral elicitation of transient evoked otoacoustic emissions, indicative of normal hearing in the right ear, and no wave $\mathrm{V}$ identification at $100 \mathrm{dBnHL}$ in the left ear. 
loss in the low and severe in the high frequencies in the female patient (not justifying any state benefit). ABR and OAEs were also indicative of normal hearing in the male patients. The female patient did not elicit OAEs (Fig. 2C), and the respective ABR curves were suggestive of mild hearing loss (Fig. 2D).

\section{Other NOHD facets}

A 50 year-old career naval officer was due to be promoted, having as a prerequisite, however, normal hearing thresholds. Clinical ENT examination was unremarkable, and the tympanometric curves were suggestive of normal middle ear ventilation. Audiometric testing was considered unreliable, due to potentially random responses to the pure tones administered (Fig. 3A). ASSR assessment proved normal in the right ear, but revealed profound hearing loss in the left ear (Fig. 3B). OAEs were also not elicited in the left ear, albeit being present in the right ear (Fig. 3C), whilst the respective ABR curves were suggestive of profound hearing loss in the left ear (Fig. 3D).

\section{Discussion}

NOHD is a multi-faceted condition encompassing various etiologies. Munchausen syndrome, malingering, or even avoidance behavior should be suspected in cases of flat pure tone audiograms, quite often inconsistent through series of examinations, provided by people with normal voice control, satisfactory communicative abilities, and obvious interests for obtaining some sort of benefit $[2,3]$.

The accurate determination of NOHD has been intensively pursued by healthcare providers in the past. The methodology employed mainly involved subjective hearing tests (i.e. Stenger test, Carhart test, Lombard voice intensity test, or lateralization test) [4], whilst ABR represented an objective examination method, which previously served as the gold standard [5]. However, not only are ASSR thresholds closer to the actual audiometric thresholds, compared to ABR testing, in the presence of hearing impairment [6], they are also superior, when the corresponding pure-tone audiogram is widely ranging between adjacent frequencies [7], or when the obtained ABR curves are not easily distinguished.

Furthermore, subjective hearing tests are often more useful in unilateral pseudo-hypacousis (i.e. Stenger test, lateralization test) [8], whilst a determined person of average ability may learn to modify his/her voice in the presence of unilateral noise, thus invalidating such tests [9]. In addition, a degree of underlying organic hearing loss could perplex the determination of the functional (non-organic) component in NOHD cases. Indeed, it is not surprising that in a large study involving 200 mil- itary candidates, the positive predictive value of the Stenger test in verifying unilateral profound hearing loss, did not exceed $87.5 \%$ [10].

The value of ASSR testing stems from the fact that it can reliably indicate the actual HLs levels in four main frequencies $(500,1,000,2,000$, and $4,000 \mathrm{~Hz})$ by obtaining a valid estimated audiogram through the use of statistical measures [11]. This is materialized, due to the physical properties of CEchirp $^{\circledR}$ sounds, which are used as auditory stimuli, which bypass the natural course of cochlear response to sound, in which the basal turn is reacting first. The CE-chirp ${ }^{\circledR}$ sounds stimulate the lower frequencies in the apical turn of the cochlea first, and through time delay subsequently the mid and basal turn of the cochlea. This results, in turn, to simultaneous cochlear firing and massive brainstem response, which is picked-up by the active electrodes, and, when mathematical prerequisites are fulfilled, is transformed to estimated HLs. More than one response from each ear can be recorded at the same time with a maximum of eight (four in each ear). Nevertheless, since most NOHD cases report profound levels of hearing impairment, it is considered safer to present one stimulus in each ear separately in order to ascertain the reliability of the obtained results.

On the other hand, ABR testing could be a useful adjunct in cases of NOHD, as it could confirm the results of the ASSR testing by adding two further advantages. The stimulus is a broad-spectrum click ranging between $2,000 \mathrm{~Hz}$ and 4,000 $\mathrm{Hz}$, hence bearing different physical properties, than the corresponding ASSR stimulus. Obtaining comparable results with different auditory stimuli confirms the soundness of NOHD diagnosis. Furthermore, the examiner is more actively participating in the diagnostic process by personally recognizing the waveform, which corresponds to the patient's HL instead of relying on a device, to perform all the necessary mathematic calculations.

Finally, the diagnosis of NOHD is completed by recording the OAEs, which represent the response of a normally functioning inner ear to an externally administered click stimulus [12]. In contrast to ASSR and ABR testing, both of which assess the acoustic output of sound (i.e. what reaches the central nervous system), OAEs assess the acoustic input of sound (i.e. what reaches the target-organ), and their presence is indicative of normal (or near-normal) cochlear function.

A non-confrontational approach should be adopted by ENT physicians, towards cases of suspected NOHD. That is because confrontation rarely leads to admission of the behavior or resolution of the problem, and this applies both for patients with a psychiatric background, and for those who are feigning hearing loss. Indeed, it is not uncommon, for many patients be- 
longing to the latter category, to be experiencing at least some degree of underlying organic hearing loss [13]. However, the certainty that ASSR testing provides, along with the confirmation from the ABR and OAE arms of the testing battery, which should be routinely performed as complementary testing for NOHD diagnosis, when a discrepancy between pure tone audiometry and ASSR results is noted and in line with the audiological principle of result cross-checking, warrants immediate discontinuation of any ENT medication and psychiatric referral in the former patients, and notification of the appropriate authorities in the latter cases. Psychiatric counselling may also be required in cases of alleged hearing adequacy when this represents a prerequisite for professional evolvement.

In conclusion, NOHD is a multi-faceted condition encompassing various etiologies. ASSR testing represents an objective and reliable method of hearing asssessment, which can serve as a gold standard in distinguishing NOHD from actual hearing loss. The use of ASSR is, therefore, strongly recommended in suspected NOHD cases, bearing medical or medico-legal implications.

\section{Acknowledgments \\ None}

\section{Conflicts of interest}

The authors have no financial conflicts of interest.

\section{Author Contributions}

Conceptualization: John Plioutas, Thomas P. Nikolopoulos. Data curation: John Plioutas, Thomas P. Nikolopoulos. Formal analysis: all authors. Funding acquisition: Thomas P. Nikolopoulos. Investigation: John Plioutas. Methodology: John Plioutas, Alexandra Vasileiou, Thomas P. Nikolopoulos. Project administration: Thomas P. Nikolopoulos, Pavlos Marangoudakis. Resources: John Plioutas, Alexandra Vasileiou, Thomas P. Nikolopoulos. Software: John Plioutas, Alexandra Vasileiou, Thomas P. Nikolopoulos. Supervision: John Plioutas, Alexandros Delidis, Thomas P. Nikolopoulos, Pavlos Marangoudakis. Validation: all authors. Visualization: Petros V. Vlastarakos, Thomas P. Nikolopoulos. Writing — original draft: John Plioutas, Petros V. Vlastarakos. Writing - review \& editing: all authors. Approval of final manuscript: all authors.

\section{ORCID iDs}

John Plioutas

Petros V. Vlastarakos

Alexandros Delidis

Alexandra Vasileiou

Thomas P. Nikolopoulos

Pavlos Maragoudakis

https://orcid.org/0000-0002-1932-1694

https://orcid.org/0000-0002-2803-1971

https://orcid.org/0000-0002-2349-2111

https://orcid.org/0000-0002-4833-8217

https://orcid.org/0000-0001-5290-1810

https://orcid.org/0000-0001-5179-5040

\section{REFERENCES}

1) Brown K, Miller J. Definition of 'deception'. The Cambridge Dictionary of Linguistics. Cambridge, UK: Cambridge University Press;2019.

2) Musat G, Sarafoleanu C, Stelea O. Hearing assesment of a malingering patient. Rom J Leg Med 2013;21:41-6.

3) Tiffany WR, Hanley CN. Delayed speech feedback as a test for auditory malingering. Science 1952;115:59-60.

4) Mehta AK, Singh VK. Screening tests for nonorganic hearing loss. Med J Armed Forces India 2000;56:79-81.

5) Stapells DR. Frequency-specific ABR and ASSR threshold assessment in young infants. In: A Sound Foundation Through Early Amplification (eds. Seewald RC, Bamford J). Stafa: Phonak;2010. p.67-105.

6) Rance G. The auditory steady-state response: generation, recording, and clinical application. San Diego: Plural Publishing;2008. p.152.

7) Vlastarakos PV, Vasileiou A, Nikolopoulos TP. The value of ASSR threshold-based bilateral hearing aid fitting in children with difficult or unreliable behavioral audiometry. Ear Nose Throat J 2017; 96:464-8.

8) Nober EH. Pseudohypacusic disturbance in adults. In: Communication Disorders (ed. Rieber RW). Boston: Springer;1981. p.275-97.

9) Goetzinger CP, Proud GO. Deafness; examination techniques for evaluating malingering and psychogenic disabilities. J Kans Med Soc 1958;59:95-101.

10) Durmaz A, Karahatay S, Satar B, Birkent H, Hidir Y. Efficiency of Stenger test in confirming profound, unilateral pseudohypacusis. J Laryngol Otol 2009;123:840-4.

11) Dimitrijevic A, John MS, Van Roon P, Purcell DW, Adamonis J, Ostroff $\mathrm{J}$, et al. Estimating the audiogram using multiple auditory steady-state responses. J Am Acad Audiol 2002;13:205-24.

12) Kemp DT. Stimulated acoustic emissions from within the human auditory system. J Acoust Soc Am 1978;64:1386-91.

13) Gelfand SA, Silman S. Functional hearing loss and its relationship to resolved hearing levels. Ear Hear 1985;6:151-8. 